\title{
Use of resting state-functional magnetic resonance imaging to assess the utility of focused meditation in post-coronavirus disease cognitive dysfunction
}

\author{
Atul Kapoor, Goldaa Mahajan, Aprajita Kapoor \\ From Consultant, Department of Radiology, Advanced Diagnostics, Amritsar, Punjab, India
}

\begin{abstract}
Post-coronavirus disease (COVID-19) syndrome is a well-recognized entity in which cognitive brain dysfunction is the most common presentation. Diagnosis and management of such patients are challenging. We describe an important brain finding of post-COVID-19 syndrome on resting (rs)-functional magnetic resonance imaging by mapping the default mode network of the brain which becomes dysfunctional thus causing patient symptoms and its correction by the technique of focused meditation.
\end{abstract}

Key words: Default mode network, Post-coronavirus disease, Resting state-functional magnetic resonance imaging

$\mathrm{C}$ oronavirus disease (COVID-19) pandemic has affected millions of people worldwide. A key concern that has emerged from it is the prevalence of neurological and longterm cognitive complications known as post-COVID syndrome [1]. Approximately, $55-75 \%$ of patients who have recovered from acute COVID-19 disease have been shown to be suffering from post-COVID syndrome with cognitive impairments affecting the quality of life [2]. Focused attention and meditation not only improve cognitive and motivational functioning (e.g. attention, mental health) but also influence the brain network's function [3]. Default mode network (DMN) is one such brain network comprising of the posterior cingulate cortex (PCC), precuneus, ventral medial prefrontal cortex (VMFC) (mPFC), temporoparietal junction (TPJ), and hippocampus [4-6]. It is significantly engaged during tasks involving social cognitive mental processes that are evaluative, including self-referential, autobiographical processing, and mentalizing, i.e. theory of mind $[7,8]$.

The purpose of this case report is to describe the use of resting state-functional magnetic resonance imaging (rs-fMRI) in mapping cognitive dysfunction in a post-COVID-19 recovered patient and its role in monitoring the effects of focused meditation using Gurbani (spiritual Sikh religion mantras) chanting on the brain networks.

\section{CASE REPORT}

A 61-year-old male doctor presented to us with complaints of diminished concentration, reduced interest, and irritability after

\section{Access this article online}

Received - 03 July 2021

Initial Review - 19 July 2021

Accepted - 28 July 2021

DOI: 10.32677/IJCR.2021.v07.i08.2983 recovery from acute post-COVID-19 disease with domiciliary self-care. He was reverse transcription-polymerase chain reaction negative and had a prior computed tomography severity score of $6 / 20$ during acute illness. There was no history of diabetes, hypertension, or any coronary artery disease.

His general physical examination along with chest skiagram was normal. His body mass index was 24.5. His Mentation Awareness Alertness Scale (MAAS) score was 2.8 and the Hospital Anxiety Depression Scale (HADS) score was 9.

rs-FMRI was done on a 1.5 Tesla Siemens Amira system using resting functional MRI protocol. Sequence parameters were as follows: Echo planar sequences were used with repetition time/ echo time $(\mathrm{TR} / \mathrm{TE})=1300 / 45 \mathrm{~ms}$, slice thickness $=4 \mathrm{~mm}$, a field of view $=256 \mathrm{~mm}$, flip angle $(\mathrm{FA})=90^{\circ}$, data matrix $=64 \times 64$, and in-plane resolution=3.5 $\mathrm{mm} \times 3.5 \mathrm{~mm}$. A high-resolution (spatial resolution: $1 \mathrm{~m}^{\mathrm{m}} 3$ ) slices, 10 min scan length structural image was also acquired using a T1-weighted magnetization prepared rapidacquisition gradient echo (MP-RAGE) pulse sequence. The data were analyzed on a Siemens resting fMRI software version 0.1.4. Region of interest analysis was done for PCC which showed hypoactivation of the VMFC along with reduced activation of bilateral temporoparietal regions of the DMN. Other networks appeared normal (Fig. 1); these findings were consistent with post-trauma stress disorder following post-COVID-19 disease.

The patient was advised to start $30 \mathrm{~min}$ of focused meditation by repetitive chanting of word Waheguru with deep inspiration and expiration repetitively with eyes closed in sitting position, 2 times a day for at least $20 \mathrm{~min}$ for a period of 6 weeks.

A follow-up was done after 6 weeks which showed his MAAS score of 5.0 with a HADS score of 5. A repeat resting fMRI

Correspondence to:

Atul Kapoor, 17/7 Kennedy avenue, The Mall Amritsar 143001, Amritsar,

Punjab, India, E-mail: masatulak@aim.com

(C) 2021 Creative Commons Attribution-NonCommercial 4.0 International License (CC BY-NC-ND 4.0). 
study was done using the prior protocol which showed complete delineation of all the nodes of the DMN, especially the medial frontal cortex where the coupling of the activity was seen with a frontoparietal network (FPN), i.e. in the dorsal lateral prefrontal cortex. There was also increased activity seen in the PCC as well as bilateral TPJ cortices (Figs. 2 a and b).

\section{DISCUSSION}

The DMN has been found to be involved in various domains of cognitive and social processing and forms one of the most important networks of the human brain. Humans spend a huge amount of time assessing one's own self and other's social relationships in space and time domain by engaging in activities such as thinking about oneself and others and reviewing past thoughts including the theory of mind. Any disruption of this network leads to severe cognitive dysfunction and has been documented in diseases such as Alzheimer, autism, and depression [8]. This case describes the impairment of DMN in post-COVID-19 disease which is the leading cause for varying degrees of cognitive dysfunction. The reduced activity in the medial frontal cortex as seen in this case leads to a lack of self-evaluation, interest, and attention, as was seen in this patient. Such findings in post-COVID-19 disease have not been described in the literature so far.

The use of focused meditation has been documented to improve cognitive function by few studies [9-11]. There are various techniques used as behavior intervention therapies such

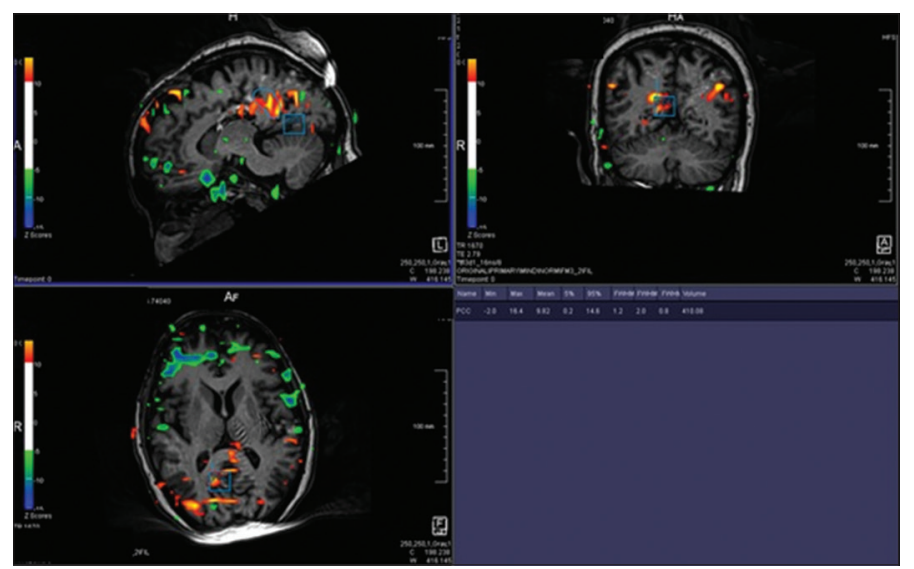

Figure 1: Baseline resting-functional magnetic resonance imaging prior to start of meditation showing absent activation of ventral medialfrontal cortex with reduced activity in bilateral temporoparietal lobes with posterior cingulate cortex
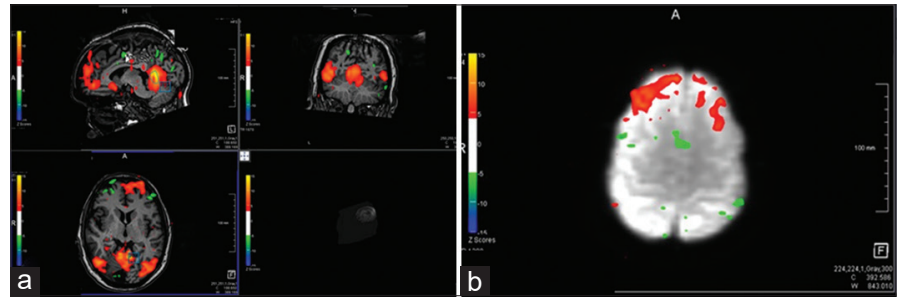

Figure 2: (a) Post-meditation study showing increased activation of medial prefrontal cortex with coupling of DACC along with increased activation of all nodes of default mode network; (b) Increased activity seen with coupling of bilateral dorsolateral parietofrontal cortices as positive and negative reinforcement and instructional therapies and various meditation methods such as mindfulness and focused meditation which has provided evidence that these therapies cause changes in activation and connectivity patterns between specific brain regions [12]. We chose focused meditation in this case as it was simple and the use of religious word chanting was more compliant with the patient which resulted in not only significant improvement of DMN pathways but also positive reinforcement of the frontolateral cortex.

Martucci et al. also showed similar results in experienced meditators with increased functional connectivity of the PCC with the dorsal ACC and dorsolateral parietofrontal cortices both during rest and meditation [12]. This was contrary to the observation of reduced FPN activity due to increased DMN activity by Kajimuro et al. [13] who showed in their study that the size of the FPN of the brain decreased and that of the DMN increased as a consequence of the meditation. Our case highlights the findings of the increased flexibility of FPN with the recruitment of the dorsal lateral frontal cortex with DMN due to the phenomenon of neuroplasticity as a result of meditation. There was a $20 \%$ increase in the regional homogeneity value in the PCC node post-meditation. Furthermore, improved coherence of both the TPJ nodes was also seen post-meditation which suggests that the type of meditation also has an influence on the degree of improved cognitive brain function.

\section{CONCLUSION}

The case highlights the role of resting functional MRI in not only diagnosing cognitive dysfunction post-COVID-19 recovery but also in the follow-up. This case demonstrates that the technique of focused meditation using chanting of Gurbani is a useful method to correct brain dysfunction.

\section{AUTHOR CONTRIBUTION}

\section{(1, 2) Design, (3), Literature.}

\section{ACKNOWLEDGMENT}

The authors would like to thank Dileep Kumar, Siemens Healthineers, Bangalore.

\section{REFERENCES}

1. Townsend L, Dyer AH, Jones K, Dunne J, Mooney A, Gaffney F, et al. Persistent fatigue following SARS-CoV-2 infection is common and independent of severity of initial infection. PLoS One 2020;15:e0240784.

2. Huang C, Huang L, Wang Y, Li X, Ren L, Gu X, et al. 6-month consequences of COVID-19 in patients discharged from hospital: A cohort study. Lancet 2021;397:220-32

3. Goldin PR, Gross JJ. Effects of mindfulness-based stress reduction (MBSR) on emotion regulation in social anxiety disorder. Emotion 2010;10:83-91.

4. Hagmann P, Cammoun L, Gigandet X, Meuli R, Honey CJ, Wedeen VJ, et al. Mapping the structural core of human cerebral cortex. PLoS Biol 2008;6:e159.

5. Buckner RL, Sepulcre J, Talukdar T, Krienen FM, Liu H, Hedden T, 
et al. Cortical hubs revealed by intrinsic functional connectivity: Mapping, assessment of stability, and relation to Alzheimer's disease. J Neurosci 2009;29:1860-73.

6. Buckner RL, Andrews-Hanna JR, Schacter DL. The brain's default network: Anatomy, function, and relevance to disease. Ann N Y Acad Sci 2008;1124:1-38.

7. Gusnard DA, Raichle ME, Raichle ME. Searching for a baseline: Functional imaging and the resting human brain. Nat Rev Neurosci 2001;2:685-94.

8. Schilbach L, Eickhoff SB, Rotarska-Jagiela A, Fink GR, Vogeley K. Minds at rest? Social cognition as the default mode of cognizing and its putative relationship to the "default system" of the brain. Conscious Cogn 2008;17:457-67.

9. Andrews-Hanna JR, Reidler JS, Huang C, Buckner RL. Evidence for the default network's role in spontaneous cognition. J Neurophysiol 2010;104:322-35.

10. Kilpatrick LA, Suyenbo BY, Smith SR, Bueller JA, Goodman T, Tillisch K, et al. Impact of mindfulness-based stress reduction training on intrinsic brain connectivity. Neuroimage 2011;56:290-8.
11. Taylor VA, Daneault V, Grant J, Scavone G, Breton E, Roffe-Vidal S, et al. Impact of meditation training on the default mode network during a restful state. Soc Cogn Affect Neurosci 2013;8:4-14.

12. Martucci F, Kraf KT, McHafe RA, Coghill RC. Neural correlates of mindfulness meditation-related anxiety relief. Soc Cogn Affect Neurosci 2013;9:751-9.

13. Kajimura S, Masuda N, King JL, Murayama K. Focused attention meditation changes the boundary and configuration of functional networks in the brain. Sci Rep 2020;10:18426.

Funding: None; Conflicts of Interest: None Stated.

How to cite this article: Kapoor A, Mahajan G, Kapoor A. Use of resting state-functional magnetic resonance imaging to assess utility of focused meditation in post-coronavirus disease cognitive dysfunction. Indian J Case Reports. 2021;7(8):351-353. 